\title{
Mediterrane Ernährung senkt das Magenkarzinom-Risiko
}

Dass neben dem Helicobacter pylori auch die Ernährungsweise bei der Manifestation eines Magenkarzinoms eine wichtige Rolle spielt, ist unbestritten. Doch welche Ernährungsfaktoren im Einzelnen positiv oder negativ wirken, ist bisher unzureichend untersucht. Nach einer neueren Untersuchung dürfte eine mediterrane Ernährung vor dem Magenkarzinom schützen.

- Im Rahmen der EPIC-Kohortenstudie wurden die Daten von 485044 Individuen im Alter zwischen 35 und 80 Jahren aus zehn europäischen Ländern erfasst und ausgewertet. Nach einer mittleren Verlaufsbeobachtung von 8,9 Jahren entwickelten 449 Patienten ein Magenkarzinom, wobei eine Korrelation zur mediterranen Ernährung nachgewiesen werden konnte, d.h. eine me- diterrane Ernährung senkte das Risiko signifikant um rel. 33\%.

\section{Kommentar}

Diese Studie ist ein weiterer Beleg dafür, dass eine mediterrane Ernährung vorteilhaft ist. Für Diabetiker und für Gefäßpatienten konnte bereits in vorangegangenen Studien eine günstige Wirkung dokumentiert werden. Nach den Ergebnissen dieser Studie kann die mediterrane Ernährung durchaus auch im Rahmen der Krebsprävention empfohlen werden, auch wenn bisher nur die Daten für das Magenkarzinom vorliegen.

P. StiefelhageN =

\begin{abstract}
- B. Buckland et al.
Adherence to a Mediterranean diet and risk of gastric adenocarcinoma within the European Prospective investigation into Cancer and Nutrition (EPIC) cohort study. Am. J. Clin. Nutr. 91 (2010) 381-390
\end{abstract}

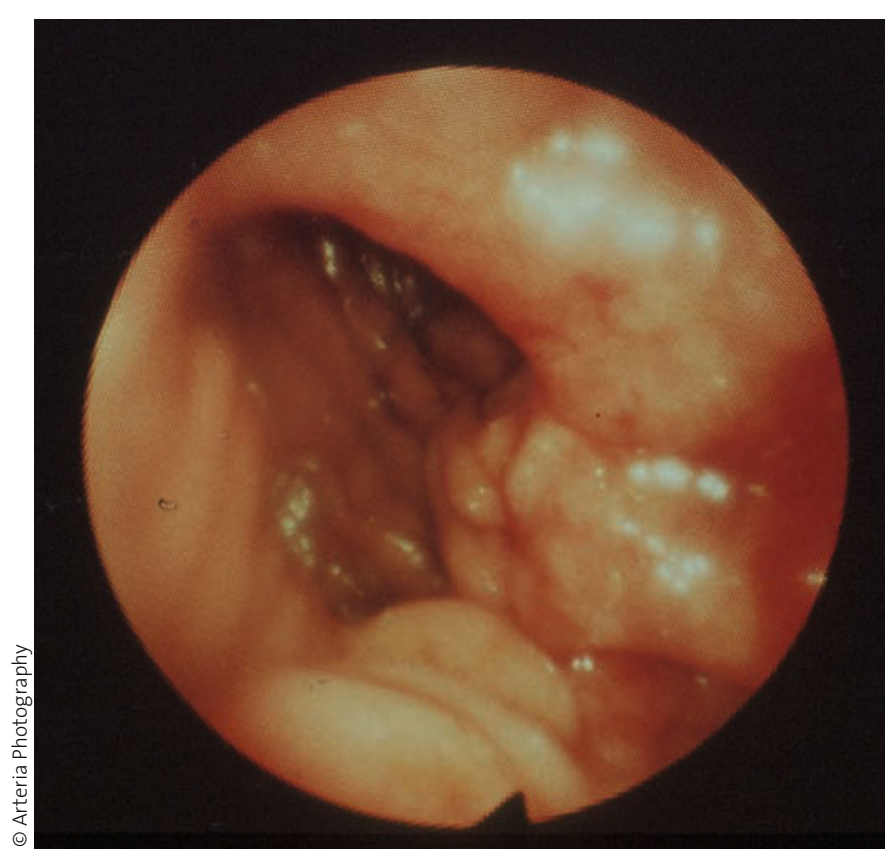

Magenkarzinom: Mediterrane Kost kann jetzt auch zur Krebsprävention empfohlen werden. 